\title{
Application of a monopole sensor for rapid in situ water quality assessment: theoretical analysis
}

\begin{abstract}
This initial study proposes a rapid and cost effective in situ water quality assessment based on reflection coefficient of a $1.749 \mathrm{~cm}$ long bare monopole senor. The reflection coefficient of the monopole sensor in air, water and ethanol were studied from measurement and calculations of Finite Element Method (FEM). FEM was used to calculate the reflection coefficient of the sensor in water/ethanol mixture solution.
\end{abstract}

Keyword: Microwave; Monopole sensor; Water contamination 